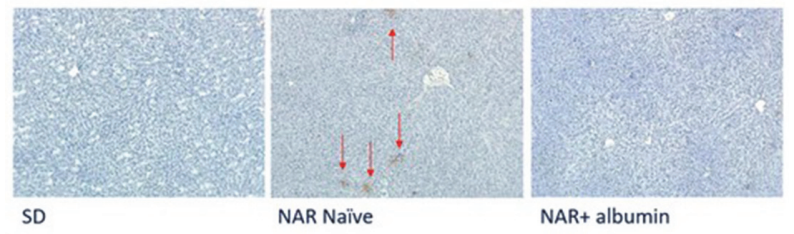

Abstract P094 Figure 1 Z0-1 immunohistochemistry of ileum showed Z0-1 was downregulated in NAR naïve group. Albumin infusion restored ZO-1 expression

albumin supplementation (figure 1). Hepatic expression of TLR4 and associated pathways: Cirrhotic NAR animals had greater hepatic TLR4 expression which was reduced by albumin administration. Hepatic TLR4 gene array confirmed the activation of TLR4 dependent pathways in the cirrhotic NAR animals, which was abrogated by albumin infusion.

Conclusion NAR animals have significantly greater liver injury, increased sensitivity to LPS and mortality which is prevented by albumin administration. Our data show for the first time that the mechanism of the protective effect of albumin is consequent upon restoration of gut junctional proteins and reduction of hepatic TLR4 expression.

\section{P095 IMPLEMENTATION OF DECOMPENSATED CIRRHOSIS DISCHARGE BUNDLE: A UNIVERSITY HOSPITAL EXPERIENCE}

Muhammad Omar Saeed*, Muhammad Zuhaid, Krishan Bountra, Unitt Esther, Nwe Ni Than. UHCW, Coventry, Coventry, UK

\subsection{6/gutjnl-2021-BASL.103}

Background Decompensated liver cirrhosis is a frequent reason for admission to acute medical and gastroenterology units. Over the last two decades, a significant rise in the prevalence of liver cirrhosis in the UK has been noted, with the major culprits being alcohol related liver diseases, hepatitis B \& C, and non-alcoholic obesity related disease. It has been observed that re-admissions to the hospital are common following discharge of the patients with decompensated liver cirrhosis. In order to improve the quality of discharge and reduce the re-admissions a decompensated discharge bundle has been developed by BSG and BASL. We aimed to assess the practice in our hospital against BSG/BASL guidelines and the impact by the implementation of the said discharge bundle.

Methods All those patients who were admitted with decompensated cirrhosis were included for data collection. Standard Quality Improvement model was adopted using two PDSA cycles. In cycle 1, discharge letters of 40 patients were assessed retrospectively against the decompensated cirrhosis discharge bundle tool kit during the months of January, February and March 2021. In cycle 2, there was re-assessment of discharge letters for 40 patients during the months of April, May and June to look for any change or improvement.

Results In cycle 1 , it was noted that only $20 \%$ of the decompensated cirrhotic patients had weight, urea and electrolytes, diuretic dose adjustment and communication with the patients regarding future plans recorded on the discharge letters. Hence, the bundle was introduced by displaying the awareness posters in the Gastro ward and discussed with the junior doctors in the board round. Additionally, emails were sent to doctors of gastro unit regarding the discharge bundle introduction.

There was a significant improvement of results in cycle 2, where $60 \%$ of the patients with decompensated cirrhosis had the above mentioned parameters documented in the discharge letters respectively.

Conclusion There were inconsistencies in the discharge letters when assessed during cycle 1 and the documentation was suboptimal. However, with the introduction of discharge bundle in the hospital has led to a significant improvement in the discharge letter documentations when compared against the decompensated cirrhosis discharge bundle in cycle 2. In order to get much better results and to continue the improvement, we would consider the incorporation of the bundle in the Trust E-Library and include in the junior doctor inductions.

\section{P096 THE POTENT UREASE INHIBITOR FLUROFAMIDE EFFECTIVELY SUPPRESSES AMMONIA PRODUCTION BY THE COLONIC MICROFLORA}

${ }^{1,2}{ }^{2}$ Saeed Motamedi, 'Robert Nelson, ${ }^{2}$ Val Edwards-Jones, 'Shaun Greer*. 'Wrightington, Wigan and Leigh Teaching Hospitals NHS Foundation Trust, Wigan, UK; ${ }^{2}$ Manchester Metropolitan University, Manchester, UK

\subsection{6/gutjnl-2021-BASL.104}

Background Ureolysis by colonic microorganisms gives rise to a significant fraction of the portal vein ammonia load; colonic urease inhibition would therefore be a logical approach to reducing systemic ammonia in patients with hepatic encephalopathy. This was trialled in the 1960's in a small number of patients, using the urease inhibitor acetohydroxamic acid (AHA); disappointing clinical results led to the approach being abandoned. However, AHA is not a particularly potent inhibitor of urease; more potent inhibitors have since been developed, one of which is flurofamide. It is possible flurofamide might be more effective in this context, but no relevant work has been reported; we have conducted an initial laboratory study into the effects of flurofamide on ammonia production by the colonic microflora, in particular the flora of the right colon.

Methods Patients attending for routine colonoscopy who did not have inflammatory bowel disease or change of bowel habit were approached to take part. At colonoscopy, $50 \mathrm{mls}$ sterile water was introduced into the right colon, and aspirated back into a polyp trap. These washings were transported to the laboratory for processing within $2-4$ hours; a mechanical cell count was performed, and the samples were incubated for 22 hours in urea-containing culture medium with various concentrations of flurofamide, under both aerobic and anaerobic conditions. Following incubation, ammonia and urea levels in the medium were measured.

Results As expected, colonic washings produced ammonia when cultured in urea containing medium, although ammonia production did not correlate directly with the number of organisms in the inoculum. Lower concentrations of flurofamide had variable effects on ammonia production, but higher concentrations reduced levels to an average of less than $5 \%$ of the ammonia generated in the absence of flurofamide (figure 1). Results from aerobic and anaerobic cultures were very similar; there was no evidence for flurofamide toxicity. Urea levels were difficult to interpret due to assay variability and the high baseline level of urea 


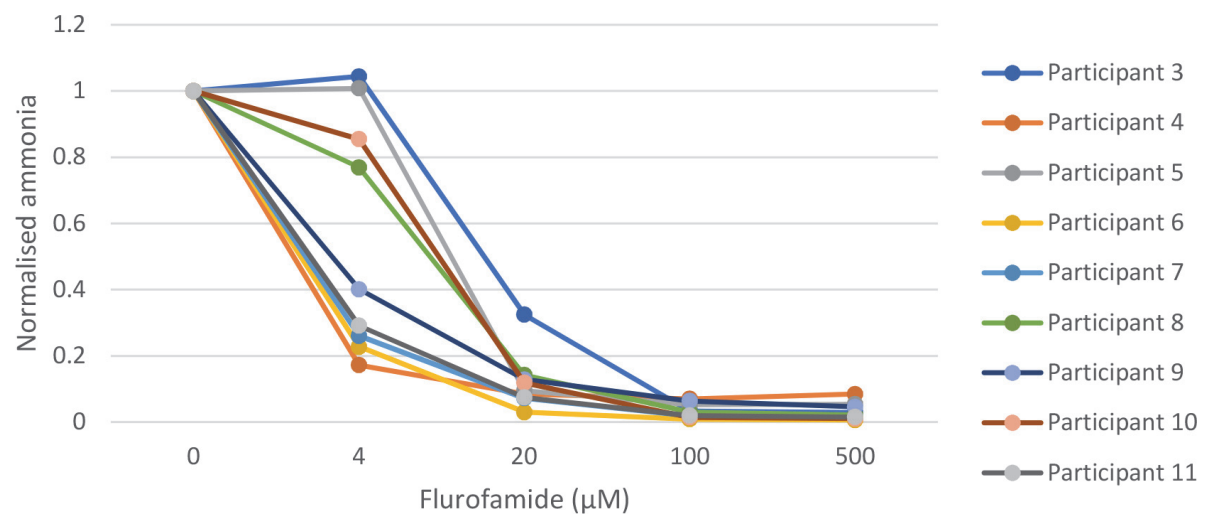

Abstract P096 Figure 1 Ammonia production, normalised to level with no flurofamide present

(300 $\mathrm{mM}$ ) in the medium used, but urea fell in cultures without flurofamide relative to cultures with higher concentrations of the compound.

Conclusion In an in vitro system, flurofamide inhibited virtually completely the production of ammonia from urea by colonic microorganisms; this occurred at concentrations which might feasibly be obtained in vivo.

\section{P097 ACUTE VARICEAL BLEEDING IN A LARGE TEACHING HOSPITAL: A 5-YEAR ANALYSIS}

Anju Phoolchund*, Megha Bhandari, Luigi Medri, Srishti Sarkar, Chanice Bloomfield, Philip Oppong, Puneet Chhabra, Philip Boger, Praful Patel, Imdadur Rahman. Southampton Interventional Endoscopy Unit, University Hospital Southampton, Southampton, UK

\subsection{6/gutjnl-2021-BASL. 105}

Acute variceal bleeding (VB) remains a major cause of death in cirrhosis/portal hypertension. We aim to assess the characteristics, therapies delivered and outcomes of patients presenting with $\mathrm{VB}$ in a large teaching hospital.

A retrospective analysis was performed on all patients presenting with acute UGIB who underwent a gastroscopy between March 2015 and February 2020. Data was collected where UGIB was deemed secondary to varices from electronic records to assess findings at endoscopy, therapy applied, rebleed rates and mortality.

A total of 2324 patients presented with UGIB. 239 presentations involving 196 patients had varices reported. 155 cases underwent therapy for acute VB as cause for UGIB, with varices felt not to be the primary cause in the remaining 84 . Median age was 54 years(IQR 46-67). 65\%(n=100) were male. Clinical presentations were haematemesis only $35 \%$ $(n=55)$, melaena only $26 \%(n=41)$ or both $38 \%(n=59)$. $56 \%$ $(n=87)$ gastroscopies were undertaken 'out of hours'. The commonest endoscopic finding was oesophageal varices in $98 \%(n=152)$.

Treatment was undertaken at first endoscopy in $93 \%$ $(\mathrm{n}=144)$, comprising banding $(86 \%)$ and glue injection(7\%). $17 \%(n=27)$ of patients required insertion of Sengstaken-Blakemore tube. The remaining $7 \%(n=11)$ underwent their first treatment at further gastroscopy (within 7 days).

$32 \%(n=49)$ of patients had repeat gastroscopy within 7 days, where $90 \%$ were due to clinical evidence of rebleed. 23 patients required repeat endoscopic therapy and 10 patients underwent Sengstaken-Blakemore tube insertion.
$30 \%(n=70)$ of patients required ICU admission. $16 \%$ $(\mathrm{n}=25)$ underwent TIPS procedure.

30 -day rebleeding rate was $24 \%(n=37)$. 30-day mortality was $17 \%(n=27)$ with $67 \%$ attributable to UGIB or subsequent decompensated cirrhosis with multi-organ failure.

On subgroup analysis, ICU admission was significantly associated with increased mortality (OR 3.46, 95\% CI 1.46 to 8.22, $\mathrm{p}<0.01$ ) but rebleeding (OR 1.35, 95\% CI 0.54 to $3.23, \mathrm{p}=0.53$ ) or having a gastroscopy 'out of hours' (OR $1.48,95 \%$ CI 0.55 to $3.95, \mathrm{p}=0.43$ ) were not.

Receiver operating characteristic (ROC) curves showed better predictive ability of MELD score than Child-Pugh score for 30-day mortality (AUC 0.92 vs 0.72 ), with MELD score of $>18$ significantly associated with increased mortality (OR $7.70,95 \%$ CI 0.04 to $0.35, \mathrm{p}<0.01$ ) similar to published data.

Mortality and rebleed rates following acute VB is in keeping with national data. More than half of patients undergo gastroscopy out of hours emphasizing the need for 24/7 access to therapeutic endoscopy. MELD score of $>18$ appears to be a strong predictor of mortality and can be used in clinical practice to consider early critical care involvement.

\section{P098 CIRRHOCARE ${ }^{\circledR}$-A PILOT STUDY OF DIGITAL HOME MANAGEMENT OF ADVANCED CIRRHOSIS TO DETERMINE FEASIBILITY AND UTILITY TO DIAGNOSE NEW DECOMPENSATION EVENTS}

${ }^{1,2}$ Konstantin Kazankov*, ${ }^{1}$ Devnandan Amor Chatterjee, ${ }^{1}$ Simone Novelli, ${ }^{1}$ Alexandra Phillips, ${ }^{3}$ Anu Balaji, ${ }^{3}$ Maruthi Raja, ${ }^{1,3}$ Rajiv Jalan, ${ }^{3}$ Ravan Boddu, ${ }^{3}$ Ravi Kumar, 1,2Rajeshwar P Mookerjee. 'Liver Failure Group, Institute for Liver and Digestive Health, UCl Medical School, Royal Free Hospital, London, UK; ${ }^{2}$ Department of Hepatology and Gastroenterology, Aarhus University Hospital, Aarhus, Denmark; ${ }^{3}$ Cyberliver Limited, Manchester, UK

\subsection{6/gutjnl-2021-BASL.106}

Background and Aims Patients discharged from hospital following acute decompensation are at high risk of new complications and need close follow-up, limited currently by the growing burden of cirrhosis and impact of COVID-19. Specialist liver care in the community is an unmet need, to reduce hospital exposure and manage new decompensation events.

Methods We included 20 patients with cirrhosis and recent acute decompensation. Commercially available devices and a smartphone (+SIM card) were given to all patients for 